\title{
Electron vortex beam generation via chiral light-induced inelastic ponderomotive scattering
}

Martin Kozák ${ }^{1, *}$

${ }^{1}$ Faculty of Mathematics and Physics, Charles University, Ke Karlovu 3, 12116 Prague 2, Czech Republic

*kozak@karlov.mff.cuni.cz

\section{Semiclassical description of the noncollinear inelastic interaction between an electron wavepacket and two light beams at different frequencies}

In the case of the noncollinear interaction between an electron wavepacket and two laser beams propagating under angles $\alpha$ and $\beta$ with respect to the electron beam propagation direction, the synchronicity condition between the electron propagation velocity and the phase velocity of the optical travelling wave is given by ${ }^{1}$ :

$$
v_{0}=\left(\omega_{1}-\omega_{2}\right) c /\left(\omega_{1} \cos \alpha-\omega_{2} \cos \beta\right)
$$

where $\omega_{1}$ and $\omega_{2}$ are the frequencies of the two light beams and $\alpha$ and $\beta$ are the angles of incidence (see Fig. S1).

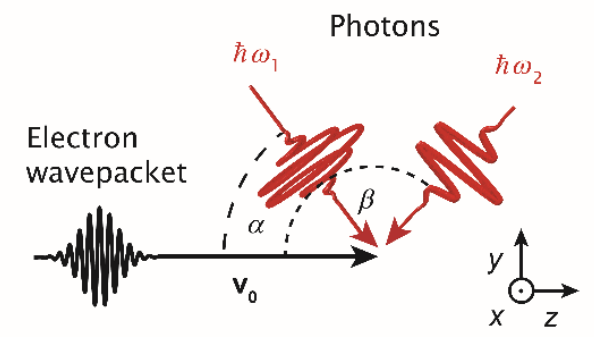

Figure S1. The geometry of the interaction of an electron wavepacket propagating with velocity $v_{0}$ with the ponderomotive potential of two optical fields at frequencies $\omega_{1}$ and $\omega_{2}$.

The semiclassical theory based on path integral representation of the wavefunction, which is used in the main manuscript, can be applied also in this case. If one of the optical beams has a form of a vortex beam, the orbital angular momentum (OAM) can be again transferred to the electron wavapacket. If we assume the coordinate transformations of light fields (we again assume linear polarization of both beams in $x$-direction) and write the fields of the two light beams in the coordinate system displayed in Fig. S1, we obtain:

$$
\begin{aligned}
& E_{1}\left(\rho_{\alpha}, z, \varphi_{\alpha}, t\right)=\frac{E_{0}}{2} g\left(t-\frac{z \cos \alpha+y \sin \alpha}{c}\right) \rho_{\alpha}^{|l|} \exp \left\{-\rho_{\alpha}^{2}-i\left[\omega_{1} t-\frac{\omega_{1}}{c}(z \cos \alpha+y \sin \alpha)\right]\right\}+i l \varphi_{\alpha} \rho_{\alpha}^{|l|}+c . c . \\
& E_{2}\left(\rho_{\beta}, z, \varphi_{\beta}, t\right)=\frac{E_{0}}{2} g\left(t-\frac{z \cos \beta+y \sin \beta}{c}\right) \exp \left\{-\rho_{\beta}^{2}-i\left[\omega_{2} t-\frac{\omega_{2}}{c}(z \cos \beta+y \sin \beta)\right]\right\}+c . c . \\
& \rho_{\alpha}=\sqrt{x^{2}+(y \cos \alpha-z \sin \alpha)^{2}} / w_{0}, \\
& \rho_{\beta}=\sqrt{x^{2}+(y \cos \beta-z \sin \beta)^{2}} / w_{0}, \\
& \varphi_{\alpha}=\arctan \left(\frac{y \cos \alpha-z \sin \alpha}{x}\right) .
\end{aligned}
$$


Here the coordinates with subscript $\alpha$ and $\beta$ refer to the coordinate systems rotated with respect to the electron propagation direction by the angles $\alpha$ and $\beta$, respectively, $E_{0}$ is the field amplitude, $g(t)$ is the temporal envelope of the pulse, $w_{0}$ is $1 / e^{2}$ radius of the beam, $r=\sqrt{x^{2}+y^{2}}, l= \pm 1$ is the OAM quantum number of the first wave and c.c. is complex conjugate. The spatially-dependent phase shift of the electron wavefunction after the interaction can be expressed using the spatio-temporal distribution of the ponderomotive potential of the synchronous wave in the electron's rest frame (coordinate transformation $z^{\prime}=z-v_{0} t, x^{\prime}=x, y^{\prime}=y$ ) as:

$$
\begin{aligned}
& \Phi\left(x^{\prime}, y^{\prime}, z^{\prime}, \varphi_{\alpha}, t\right)=\frac{1}{\hbar} \int_{t_{0}}^{t}\left\{\frac{e^{2} E_{0}^{2}}{4 m \omega_{1} \omega_{2}} g\left[t^{\prime}-\frac{\left(z^{\prime}+v_{0} t^{\prime}\right) \cos \beta+y^{\prime} \sin \beta}{c}\right] g\left[t^{\prime}-\frac{\left(z^{\prime}+v_{0} t^{\prime}\right) \cos \alpha+y^{\prime} \sin \alpha}{c}\right] \times\right. \\
& \left.\times \rho_{\alpha}^{|l|} \exp \left[-\rho_{\alpha}^{2}-\rho_{\beta}^{2}+i\left(\frac{\omega_{1} \cos \alpha-\omega_{2} \cos \beta}{c} z^{\prime}+\frac{\omega_{1} \sin \alpha+\omega_{2} \sin \beta}{c} y^{\prime}\right)+i l \varphi_{\alpha}\right]+c . c .\right\} d t^{\prime}= \\
& =\alpha_{\mathrm{nc}} \cos \left(\frac{\omega_{1} \cos \alpha-\omega_{2} \cos \beta}{c} z^{\prime}+\frac{\omega_{1} \sin \alpha+\omega_{2} \sin \beta}{c} y^{\prime}+l \varphi_{\alpha}\right)
\end{aligned}
$$

where the coupling constant $\alpha_{\mathrm{nc}}$ equals to:

$$
\begin{aligned}
& \alpha_{\mathrm{nc}}\left(x^{\prime}, y^{\prime}, z^{\prime}\right)=\frac{e^{2} E_{0}^{2}}{2 m \omega_{1} \omega_{2} \hbar} \int_{t_{0}}^{t}\left\{g\left[t^{\prime}-\frac{\left(z^{\prime}+v_{0} t^{\prime}\right) \cos \beta+y^{\prime} \sin \beta}{c}\right] g\left[t^{\prime}-\frac{\left(z^{\prime}+v_{0} t^{\prime}\right) \cos \alpha+y^{\prime} \sin \alpha}{c}\right] \times\right. \\
& \left.\times \rho_{\alpha}^{|l|} \exp \left(-\rho_{\alpha}^{2}-\rho_{\beta}^{2}\right)\right\} d t^{\prime}
\end{aligned}
$$

The coupling constant, which determines the strength of the interaction for the given initial transverse coordinates of the electron wavepacket $x^{\prime}$ and $y^{\prime}$, depends now not only on the distance from the symmetry axis of the electron beam $\rho$ and on the longitudinal shape of the envelopes of the two laser beams, but there is a complex coupling between the transverse and the longitudinal properties of the light fields due to nonzero angles of incidence. For the known envelopes of the pulses, the integral in Eq. (S4) can be calculated numerically (we again assume the initial electron wavefunction in the form of a plane wave and negligible change of electron velocity during the interaction, which allows to integrate along the classical electron trajectory in $z$ direction).

When the widths of the laser beams $w_{0}$ are much larger than the spatial period of the longitudinal phase modulation of the electron wavepacket $\Lambda=2 \pi c /\left(\omega_{1} \cos \alpha-\omega_{2} \cos \beta\right)$, the coupling constant changes slowly compared to the fast oscillating phase term in the result of Eq. (S3), which determines the phase properties of the electron wavepacket. The spectral decomposition to different photon orders can be done similarly to the case of the counterpropagating laser pulses using Eq. (6). The phase profiles of the resulting vortex states are tilted with respect to the electron propagation direction. The tilt corresponds to the fact that the AOM vector has nonzero transverse component ${ }^{2,3}$, which be controlled by the experimental geometry and the parameters of the two light beams. This is in agreement with the expectations from the particle picture of the noncollinear interaction because the vector of OAM of the vortex photon is not parallel to the electron beam.

\section{References:}

(1) Kozák, M.; Eckstein, T.; Schönenberger, N.; Hommelhoff, P. Inelastic Ponderomotive Scattering of Electrons at a High-Intensity Optical Travelling Wave in Vacuum. Nat. Phys. 2018, 14 (2), 121-125. https://doi.org/10.1038/nphys4282.

(2) Aiello, A.; Banzer, P.; Neugebauer, M.; Leuchs, G. From Transverse Angular Momentum to Photonic Wheels. Nat. Photonics 2015. https://doi.org/10.1038/nphoton.2015.203.

(3) Bliokh, K. Y.; Nori, F. Transverse and Longitudinal Angular Momenta of Light. Physics Reports. 2015. https://doi.org/10.1016/j.physrep.2015.06.003. 